\title{
Ovarian-adrenal cross-talk in polycystic ovary syndrome: evidence from wedge resection
}

\author{
Xiao-Ke Wu ${ }^{1,2}$, Shan-Ying Zhou ${ }^{1}$, Kirsimarja Sallinen ${ }^{2}$, Pasi Pöllänen ${ }^{2}$ and Risto Erkkola ${ }^{2}$ \\ ${ }^{1}$ Department of Obsterics and Gynaecology, Jinling Hospital of Nanjing University, Nanjing 210002, China and \\ ${ }^{2}$ Department of Obstetrics and Gynaecology, University Central Hospital of Turku, Turku 20520, Finland \\ (Correspondence should be addressed to X-K Wu, Department of Obstetrics and Gynaecology, University Central Hospital of Turku, \\ Turku 20520, Finland; Email: xiaoke.wu@utu.fi)
}

\begin{abstract}
Objective: To determine whether the ovary influences adrenal androgen secretion in women with polycystic ovary syndrome (PCOS).

Design: Six PCOS-affected patients with clomiphene resistance and gonadotrophin hyperresponsivity, and six controls with regular ovulatory cycles, matched for age and body mass index.

Methods: Bilateral ovarian wedge resection was performed to induce ovulation surgically for these refractory women with PCOS. The adrenal androgen secretions were evaluated in PCOS patients before and again 6 months after this surgery, and in the controls, using an ACTH stimulation test $(0.25 \mathrm{mg}$ synthetic ACTH(1-24)).

Results: Biochemically, basal levels and the maximum net increases $(\Delta)$ of 17-hydroxyprogesterone (17-OHP) and androstenedione, $\Delta 17-\mathrm{OHP} / \Delta$ progesterone and $\Delta$ androstenedione/ $\Delta 17-\mathrm{OHP}$ ratios in response to exogenous ACTH were significantly higher in PCOS patients before operation than those of controls. This purely ovarian surgery in women with PCOS was found to significantly reduce their basal androstenedione, testosterone and LH levels, insulin/glucose ratio, and post-corticotrophic $\Delta 17-\mathrm{OHP}, \Delta$ androstenedione, $\Delta 17-\mathrm{OHP} / \Delta$ progesterone and $\Delta$ androstenedione/ $\Delta 17-\mathrm{OHP}$, without obvious changes in FSH, oestradiol, sex hormone-binding globulin, $\Delta$ dehydroepiandrosterone, $\Delta$ dehydroepiandrosterone sulphate, $\Delta$ aldosterone and $\Delta$ cortisol values.

Conclusions: Ovarian hyperandrogenicity from polycystic ovary may contribute to the enhanced adrenal P450c17 $\alpha$ activity and subsequent $\Delta^{4}$ androgen reserve revealed by the pharmacological corticotrophin stimulation in our special PCOS cases.
\end{abstract}

European Journal of Endocrinology 143 383-388

\section{Introduction}

Increased androgen production is the biochemical hallmark of polycystic ovary syndrome (PCOS), and is central to the pathogenesis of the morphological changes in the ovaries and the clinical syndrome. Numerous investigators have found elevated androgen synthesis by the adrenal gland in patients with this syndrome. Both basal levels of dehydroepiandrosterone sulphate (DHEAS) and 11 $\beta$-hydroxyandrostenedione, the exclusive products of the adrenal, and responses of adrenal androgens to adrenocorticotrophic hormone (ACTH) have been found to be elevated in $70-75 \%$ of affected patients, independent of their ethnic diversity (1). Adrenal hyperandrogenism, therefore, constitutes a main endocrine component of PCOS.

Adrenal enzyme dysfunction characterized by a deficiency is a relatively rare phenomenon, which has many biochemical and clinical overlaps with PCOS, and should be differentiated from this entity $(1,2)$. In contrast, enhanced adrenal enzymatic activity has been proposed to occur in association with PCOS. Some of the adrenal enzymes which explain this association between PCOS and adrenal hyperandrogenism include both arms of the cytochrome P450c17 $\alpha$ enzyme, 17-hydroxylase and 17,20-lyase, as well as $11 \beta$ hydroxysteoid dehydrogenase, etc. $(3,4)$.

Recently, available data from treatment with gonadotrophin-releasing hormone (GnRH) agonists and insulin (INS)-sensitizing drugs in PCOS have further suggested that ovary functionality and INS resistance may be involved in the altered adrenal enzyme activity and subsequent steroid production (4-6). To our knowledge, there have been no studies evaluating the effect of bilateral ovarian wedge resection (OWR) on steroidogenic responses of the adrenal gland in PCOS women of Asian background, especially with failed drug ovulation induction. We hypothesized 
that ovarian androgen excess, a main endocrine feature in this syndrome, may contribute to adrenal dysfunction in PCOS.

\section{Materials and methods}

\section{Subjects}

The University Ethics Committee approved this study, and informed consent was obtained from each subject. Six PCOS-affected women with both clomiphene resistance and gonadotrophin hyperreponsivity were selected for the study. Before OWR, they all exhibited the classic features of oligomenorrhoea, anovulation, elevated total testosterone levels $(>2.5 \mathrm{nmol} / \mathrm{l})$ and a ratio of luteinizing hormone $(\mathrm{LH})$ to follicle-stimulating hormone (FSH) greater than 2. Enlarged polycystic ovaries containing multiple small follicles and increased stroma were confirmed by transvaginal sonography and diagnostic laparoscopy. Their mean ( \pm S.D.) age was $27.4 \pm 4.3$ years (range, 24-31 years) and their mean ( \pm S.D.) body mass index (BMI) was $21.9 \pm 2.2 \mathrm{~kg} / \mathrm{m}^{2}$. Six women with normal ovulation, four of whom previously had tubal infertility and two of whom had infertility due to a male factor, were recruited as the control group because they demonstrated a biphasic basal body temperature and a luteal phase progesterone level of $>19.2 \mathrm{nmol} / \mathrm{l}$. The mean age and BMI for these controls was $26.8 \pm 4.7$ years (range, $23-30$ years) and $22.6 \pm 1.7 \mathrm{~kg} / \mathrm{m}^{2}$ respectively.

Clomiphene resistance was defined as the absence of follicular development for three cycles on transvaginal ultrasonagraphic examination with concomitant failure of oestradiol $\left(\mathrm{E}_{2}\right)$ levels to rise after treatment with clomiphene citrate, $150 \mathrm{mg}$, for 5 days. Six patients who met the inclusion criteria were further determined to be gonadotrophin hyperresponsive. Three patients had a previous cycle complicated by moderate ovarian hyperstimulation syndrome according to the modified criteria (7). The other three patients had a previous ovulation induction with human gonadotrophin cancelled because of hyperresponsivity $\left(\mathrm{E}_{2}\right.$ level $>9.18 \mathrm{nmol} / \mathrm{l}$ or more than four follicles of $>20 \mathrm{~mm}$ in average diameter with a large number of small to intermediate-sized follicles). Only diagnostic laparoscopy was available at that time in our department and these patients refused a trial treatment because of no experience by the physicians. As the last step for their long-lasting anovulatory infertility, bilateral OWR was performed in these refractory patients with PCOS in the manner described previously (8).

Since obesity is a well-recognized cause of either resistance or hyperresponsiveness to drug ovulation induction, only non-obese patients were enrolled (7). None of the subjects in either group was obese (BMI $<25 \mathrm{~kg} / \mathrm{m}^{2}$ ) or had taken any medication, including hormonal contraception for the previous 3 months. Subjects in both groups were matched for age and BMI.
The waist/hip ratio (WHR), a simple anthropometric method, was further used to determine the pattern of body fat distribution. The waist and hip circumferences were measured as previously described (9). Thyroid dysfunction, hyperprolactinaemia, congenital adrenal hyperplasia $(\mathrm{CAH})$ and androgen-secreting tumours were specifically excluded by appropriate tests.

\section{Protocol}

The ACTH test was performed in the midfollicular phase (days 5-7) of the cycle for menstruating subjects (such as all controls and the PCOS patients after OWR). In cases where patients with PCOS were amenorrhoeic before operation, data were collected when the serum progesterone level was $<8.0 \mathrm{nmol} / \mathrm{l}$. Days 5-7 were chosen on the basis of observations that there is no difference in the adrenocortical response to acute ACTH stimulation between tests arranged on days 3-5 and tests arranged on days 6-8 of the menstrual cycle (10). All women were asked to attend the hospital at $08.00 \mathrm{~h}$ after an overnight fast, and i.v. cannulas with heparin locks were placed in antecubital veins, followed by a $30 \mathrm{~min}$ rest to minimize the effects of physical activity and non-specific stress. Immediately after the baseline blood collection, all women received an i.v. bolus of $0.25 \mathrm{mg}$ synthetic ACTH(1-24) (Biochemical Company, Shanghai, China) over $60 \mathrm{~s}$, and blood was sampled every $30 \mathrm{~min}$ for $2 \mathrm{~h}$. Then serum was separated and stored at $-80{ }^{\circ} \mathrm{C}$ until assayed. In PCOS patients, this adrenal dynamic test was carried out at an appropriate date before the OWR operation (PCOS-BO patients) and repeated 6 months after the operation (PCOS-AO patients), while in controls ACTH was administered once only. Dexamethasone was not given to any women of the three groups on the evening before the study day, in order to assess the resting basal steroid levels.

\section{Determinations}

Using commercially available kits, serum responsive levels of FSH, LH, $\mathrm{E}_{2}$, progesterone, testosterone, aldosterone (ALD), cortisol, INS (kits from NT Biomedical, Tianjin, China), androstenedione, dehydroepiandrosterone (DHEA), DHEAS (kits from Diagnostic Products Corporation, Los Angeles, CA, USA), and 17-hydroxyprogesterone (17-OHP) (kits from Biosource, Fleurus, Belgium) during the ACTH test, as well as basal sex hormone-binding globulin (SHBG) (kits from Wallac Oy, Turku, Finland), glucose and endogenous ACTH (kits from Amersham, Arlington Heights, IL, USA) were evaluated in all subjects of the three groups with welldetermined procedures $(8-10)$. The intra- and interassay precision coefficients of variation were 2.3 and $7.5 \%$ for $\mathrm{LH}, 2.5$ and $8.7 \%$ for FSH, 7.4 and $6.1 \%$ for testosterone, 9.4 and $14.8 \%$ for androstenedione, 8.0 and $7.7 \%$ for $\mathrm{E}_{2}, 7.2$ and $8.9 \%$ for progesterone, 3.4 and 
$6.7 \%$ for INS, 8.6 and $7.3 \%$ for cortisol, 6.6 and $7.9 \%$ for ALD, 4.4 and $7.4 \%$ for 17-OHP, 5.3 and $6.8 \%$ for DHEA, 9.8 and $9.5 \%$ for DHEAS, 4.3 and $6.5 \%$ for SHBG, and 6.9 and $7.9 \%$ for ACTH. All samples from the same patients were assayed in the same batch. Anthropometric data (BMI and WHR) were also obtained just before the adrenal dynamic test in each woman of the three groups.

\section{Statistics and calculations}

The extra-adrenal contribution to circulating levels of adrenal products could affect the evaluation of the response to ACTH. To avoid this, the analysis of the release of steroids after the exogenous ACTH stimulation test was performed on data normalized as net increase over basal values. The maximum net increases $(\Delta)$ over baseline values in terms of androstenedione, DHEA, and DHEAS after this pharmacological ACTH were calculated to evaluate the adrenal androgen reserve. The activities of $17 \alpha$-hydroxylase and 17,20lyase were represented by the mean ratios of $\Delta 17-\mathrm{OHP}$ to $\Delta$ progesterone $(\Delta 17-\mathrm{OHP} / \Delta$ progesterone $)$ and $\Delta$ androstenedione to $\Delta 17-\mathrm{OHP}$ ( $\Delta$ androstenedione/ $\Delta 17-\mathrm{OHP})$ respectively. INS resistance was assessed by the ratio of fasting INS $(\mathrm{mU} / \mathrm{l})$ to fasting glucose $(\mathrm{mmol} / \mathrm{l})$, a simple and accurate indicator as reported by Legro et al. (11). The results are reported as means \pm s.D. In women with PCOS, we compared the results before operation with those after operation by using the Wilcoxon signed-rank test. Between the PCOS and control subjects the results were compared by using the Mann-Whitney U test. Pearson's product moment correlation was used to estimate linear relationships between variables. $P<0.05$ was regarded as statistically significant.

\section{Results}

The clinical and basal hormonal characteristics of PCOS and control subjects are shown in Table 1. As compared with controls, PCOS-BO patients demonstrated significantly higher mean serum levels of LH, testosterone, androstenedione, 17-OHP and INS, while BMI, WHR and serum FSH, $\mathrm{E}_{2}$, progesterone, glucose, ALD, DHEA, SHBG, ACTH and cortisol levels were similar, resulting in higher mean LH/FSH and INS/ glucose ratios. As for PCOS-AO patients, OWR significantly reduced their mean levels of testosterone, androstenedione, 17-0HP, LH and INS without obvious changes in BMI, WHR, FSH, $\mathrm{E}_{2}$, progesterone, glucose, ALD or cortisol, followed then by normal LH/FSH and INS/glucose ratios. Furthermore, no significant variations were found in subjects of the three groups for mean $\mathrm{LH}, \mathrm{FSH}, \mathrm{E}_{2}$ and testosterone levels in response to the ACTH stimulation test, with persistently higher $\mathrm{LH}$ and testosterone values in PCOS-BO patients than in the other two groups (data not shown).

Figure 1 summarizes the maximum net incremental responses of INS and some adrenal steroids during adrenal dynamic tests in the three groups. After ACTH stimulation, no significant differences existed among the three groups for $\Delta$ progesterone, $\triangle$ DHEA, $\triangle$ DHEAS, $\Delta$ cortisol and $\Delta$ ALD. However, PCOS-BO patients

Table 1 Clinical and basal hormonal characteristics (means \pm S.D.) of PCOS patients before (PCOS-BO) and after (PCOS-AO) bilateral OWR, and in control subjects.

\begin{tabular}{|c|c|c|c|}
\hline \multirow[b]{2}{*}{ Determination } & \multicolumn{2}{|c|}{$\operatorname{PCOS}(n=6)$} & \multirow[b]{2}{*}{ Controls $(n=6)$} \\
\hline & $\mathrm{BO}$ & $\mathrm{AO}$ & \\
\hline BMI $\left(\mathrm{kg} / \mathrm{m}^{2}\right)$ & $21.9 \pm 2.2$ & $21.8 \pm 2.3$ & $22.6 \pm 1.7$ \\
\hline WHR & $0.80 \pm 0.06$ & $0.81 \pm 0.06$ & $0.81 \pm 0.05$ \\
\hline Testosterone (nmol/l) & $4.78 \pm 0.42^{\star *}$ & $2.27 \pm 0.36 \dagger$ & $2.08 \pm 0.38$ \\
\hline Androstenedione (nmol/l) & $13.9 \pm 2.5^{\star \star}$ & $9.0 \pm 1.8 \dagger$ & $8.8 \pm 1.7$ \\
\hline $\mathrm{E}_{2}(\mathrm{pmol} / \mathrm{l})$ & $341 \pm 44$ & $365 \pm 49$ & $334 \pm 66$ \\
\hline Progesterone (nmol/l) & $0.99 \pm 0.32$ & $0.95 \pm 0.45$ & $0.92 \pm 0.29$ \\
\hline 17-OHP (nmol/l) & $3.5 \pm 0.15^{\star *}$ & $2.0 \pm 0.36 \dagger$ & $1.4 \pm 0.9$ \\
\hline $\mathrm{DHEA}(\mu \mathrm{g} / \mathrm{l})$ & $8.3 \pm 1.9$ & $9.5 \pm 2.2$ & $8.4 \pm 2.3$ \\
\hline DHEAS $(\mu \mathrm{mol} / \mathrm{l})$ & $8.0 \pm 1.9$ & $9.0 \pm 2.6$ & $8.5 \pm 1.8$ \\
\hline Cortisol $(\mu \mathrm{g} / \mathrm{dl})$ & $8.7 \pm 2.5$ & $8.3 \pm 2.6$ & $6.7 \pm 0.8$ \\
\hline ALD $(p g / l)$ & $10.6 \pm 1.7$ & $10.4 \pm 1.2$ & $9.5 \pm 1.4$ \\
\hline SHBG (nmol/l) & $42.1 \pm 9.7$ & $51.6 \pm 8.8$ & $47.8 \pm 8.7$ \\
\hline LH (IU/I) & $15.7 \pm 3.7^{\star \star}$ & $8.8 \pm 1.9 \dagger \dagger$ & $8.0 \pm 0.9$ \\
\hline FSH (IU/I) & $6.1 \pm 1.0$ & $4.9 \pm 1.8$ & $4.5 \pm 0.9$ \\
\hline LH/FSH & $3.3 \pm 0.61^{*}$ & $1.1 \pm 0.33 † \dagger$ & $1.3 \pm 0.21$ \\
\hline ACTH (ng/l) & $15.6 \pm 2.3$ & $14.9 \pm 3.4$ & $16.0 \pm 2.9$ \\
\hline INS (mU/I) & $12.9 \pm 1.4^{*}$ & $9.0 \pm 2.1 \dagger$ & $8.1 \pm 1.7$ \\
\hline Glucose $(\mathrm{mmol} / \mathrm{l})$ & $4.2 \pm 0.38$ & $4.4 \pm 0.33$ & $4.5 \pm 0.35$ \\
\hline Insulin/glucose (U/mol) & $2.9 \pm 0.3^{\star \star}$ & $2.0 \pm 0.3 \dagger$ & $2.0 \pm 0.2$ \\
\hline
\end{tabular}

${ }^{*} P<0.05,{ }^{* \star} P<0.01$ vs controls; $\uparrow P<0.05, \dagger \dagger P<0.01$ vs PCOS-BO. 

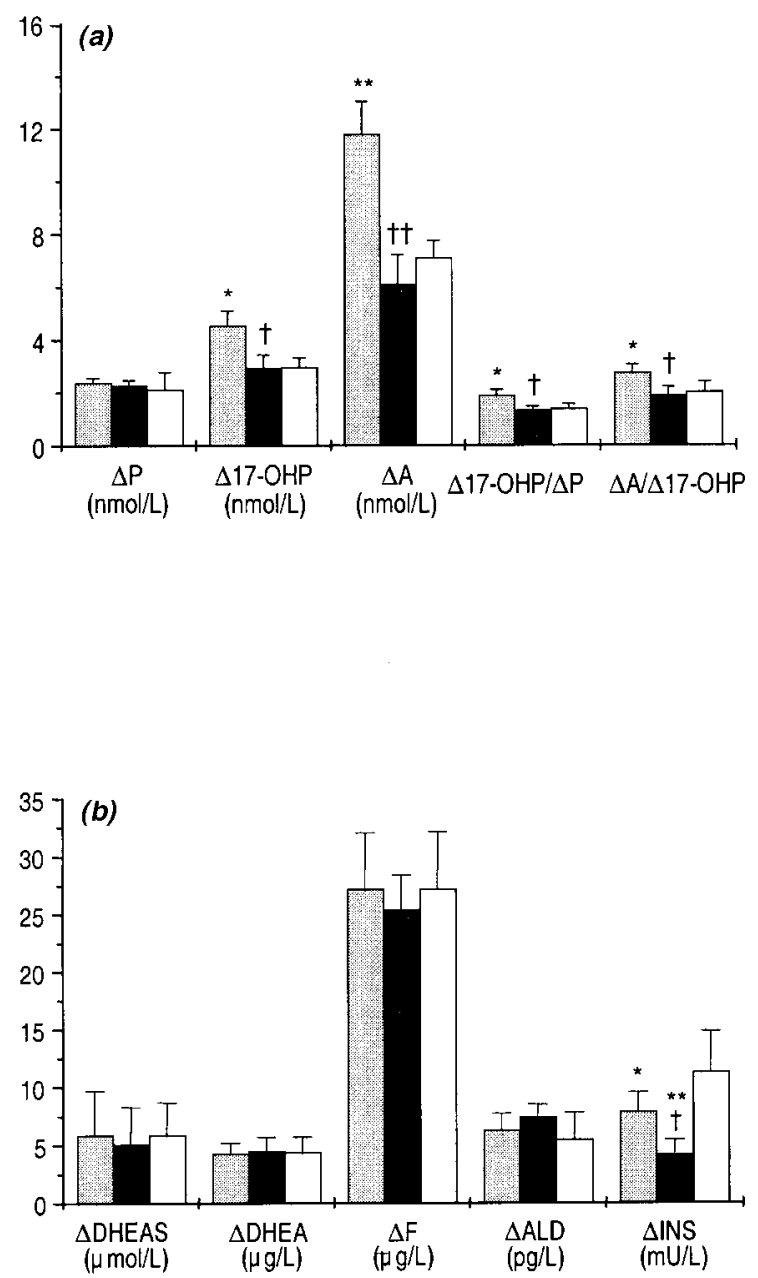

Figure $1(a)$ The maximum net increase $(\Delta)$ of progesterone $(P)$, 17-OHP and androstenedione $(\mathrm{A})$ and the ratios $\Delta 17-\mathrm{OHP} / \Delta \mathrm{P}$ and $\Delta \mathrm{A} / \Delta 17-\mathrm{OHP}$; and $(b) \Delta$ of DHEAS, DHEA, cortisol (F), ALD and INS during the ACTH stimulation test in PCOS-BO (shaded columns) and PCOS-AO (filled columns) patients and controls (open columns). ${ }^{\star} P<0.05,{ }^{\star \star} P<0.01$ vs controls; ${ }^{\dagger} P<0.05,{ }^{\ddagger} P<0.01$ vs $\mathrm{PCOS}-\mathrm{BO}$ patients.

demonstrated much higher mean post-corticotrophic $\Delta 17-\mathrm{OHP}$ and $\Delta$ androstenedione, and their ratios, $\Delta 17-\mathrm{OHP} / \Delta$ progesterone and $\Delta$ androstenedione $/ \Delta 17-$ $\mathrm{OHP}$, with respect to controls. OWR modified the postcorticotrophic $\Delta 17-\mathrm{OHP}$ and $\Delta$ androstenedione secretions, and $\Delta 17-0 \mathrm{HP} / \Delta$ progesterone and $\Delta$ androstenedione/ $\Delta 17-\mathrm{OHP}$ ratios in women with $\mathrm{PCOS}$, as the differences of these indices disappeared between PCOS-AO patients and controls. INS levels were elevated during ACTH stimulation in all subjects of the three groups, the net responses being higher in controls, lower in PCOS-BO patients. The surgical approach of OWR reduced the ACTH-induced $\triangle \mathrm{INS}$ in women with PCOS. Linear regression analysis found positive correlations between $\Delta \mathrm{INS}$ and $\Delta 17-\mathrm{OHP} / \Delta$ progesterone $(r=0.62, P<0.05)$, and between $\Delta \mathrm{INS}$ and
$\Delta$ androstenedione/ $\Delta 17-\mathrm{OHP}(r=0.47, P<0.05)$, when all data for both PCOS-BO and PCOS-AO patients were put together as a group.

\section{Discussion}

Clomiphene citrate is an oestrogen antagonist that is widely used to induce ovulation by competing oestrogen receptors at the hypothalamic level, while gonadotrophins work directly on the ovarian receptors for follicle development. The mechanism of anovulation behind both clomiphene-resistant and gonadotrophinhyperresponsive patients with PCOS in the present study seems to lie at the level of a defective pituitary gland. Our findings that reduced androgen levels after the bilateral OWR were associated with appropriate gonadotrophin sections and then spontaneous ovulation suggest a detrimental effect of androgens on the pituitary release of gonadotrophins and ovarian performance of normal functionality (12). On the other hand, it seems likely that adrenal hyperandrogenism is acting to maintain the ovulatory dysfunction in some patients with PCOS. Approximately $50-70 \%$ of PCOS patients have excessive adrenal androgen levels and treatment with glucocorticoids only has been reported to improve menstrual regularity in 30-66\% of oligo-ovulatory patients (13). Further, corticosteroid suppression may enhance the ovulatory response to clomiphene citrate, particularly in those patients with higher DHEAS levels (14). Although no differences were found for basal levels and maximum net increases of DHEA and DHEAS among three groups, higher $\Delta$ androstenedione in response to ACTH stimulation in PCOS-BO patients suggests its adrenal origin. As far as we know, no previous studies have evaluated the adrenal function in such special cases with PCOS.

The ACTH stimulation test can be performed in several ways depending on the intended purpose. If a pharmacological dose of ACTH is administered without pretreatment with dexamethasone, the maximal incremental rise between the pre- and post-stimulation values of the various corticosteroids is an indicator of adrenal reserve. In hyperandrogenic women the ACTH stimulation test is able to show changes in adrenal secretion as well as subtle enzymatic alterations that a basal hormonal evaluation fails to show (15). Thirty- or 60-min ACTH stimulation tests have been widely used for the diagnosis of late-onset $\mathrm{CAH}$ or heterozygote carriers of CAH. It has been suggested that it is possible to evaluate adrenal steroid production by determining serum cortisol and 17-OHP levels with longer time intervals. Recently, it has also been reported that steroid levels reach a plateau 60-90 min after ACTH stimulation (10). Therefore, we extended blood sampling time to $120 \mathrm{~min}$ with $30 \mathrm{~min}$ intervals to observe the long-term changes in serum progesterone, 17-OHP levels and androstenedione as well as 17-OHP/ progesterone and androstenedione/17-OHP ratios. In 
the present study, before operation women with PCOS demonstrated significantly higher post-stimulation $\Delta 17-\mathrm{OHP}$ and $\Delta$ androstenedione, and higher ratios of $\Delta 17-\mathrm{OHP} / \Delta$ progesterone and $\Delta$ androstenedione $/ \Delta 17$ $\mathrm{OHP}$, indicating a greater androgen reserve in terms of androstenedione. Our results are in accord with data (16) in which only a physiological ACTH dose was used instead of the pharmacological amount for stimulation to evaluate adrenal cytochrome $\mathrm{P} 450 \mathrm{c} 17 \alpha$ activity, and are at variance with a study (17) which failed to find enhanced activity of this key limiting enzyme in adrenal steroidogenesis.

As shown in this study, hyperinsulinaemia and INS resistance is another component of the endocrine pattern of PCOS and may have a pathogenic role for the adrenal alteration $(1,5)$. Furthermore, in patients with PCOS, positive correlations were found after ACTH stimulation between maximum net INS changes and $\Delta 17-\mathrm{OHP} / \Delta$ progestereone and $\Delta$ androstenedione $/ \Delta 17$ OHP ratios. These data suggest an important influence of INS on adrenal $\Delta^{4}$ androgen production, probably by the activation of both arms of $\mathrm{P} 450 \mathrm{c} 17 \alpha$, namely 17-hydroxylase and 17,20-lyase. Furthermore, metformin therapy in some PCOS patients has been reported to improve INS sensitivity and reduce the basal and post-glucose load INS levels as well, but it fails to alter 17-OHP and androstenedione responses to ACTH and cytochrome P450c17 $\alpha$ activity (18). Ethnic and presumably underlying genetic differences in INS resistance may explain such a finding, as metformin appears effective for androgen suppression in certain populations, such as Venezuelan (19) and Finnish (20), but ineffective in others, such as American (21) and Turkish (22). It has been stated that an INS-sensitizing agent is effective for endocrine abnormalities and ovulatory performance in PCOS women of Asian background (23). Therefore, therapeutic roles of INSlowering drugs for adrenal enzyme alteration and steroid biosynthesis warrant further investigation in the future.

Many studies have been dedicated to evaluating the possibility that altered ovarian function is the cause of adrenal androgen excess in PCOS. GnRH agonists, which inhibit the ovary selectively, without discernible direct effects on adrenal function, have been used to suppress ovarian activity (24). Indeed, studies with GnRH administration have shown a reduction of adrenal androgens and adrenal androgen response to ACTH in some PCOS patients $(6,16)$. Among the ovarian factors which may be involved, hyperoestrogenism and androgen excess have attracted most attention. Recently, it has been shown that oestrogen administration to PCOS women treated with GnRH agonist results in an enhancement of some adrenal enzymatic activities (6). More recently, in PCOS patients, serum unbound $\mathrm{E}_{2}$ has also been demonstrated to correlate positively with basal serum DHEAS, DHES and 17hydroxypregnenolone (25). This suggests a positive influence of oestrogens on the $\Delta^{5}$ pathway, predominantly resulting in an enhancement of DHEA and DHEAS production. In contrast, we were not able to demonstrate any differences in basal $\mathrm{E}_{2}$ level, or basal values and post-corticotrophic maximum net increases of DHEA and DHEAS between PCOS-BO patients and controls. It seems unlikely that oestrogen alone is sufficient to cause adrenal hyperandrogenism here. Conversely, our work on purely ovarian surgery by OWR, showing decreased ovarian testosterone and androstenedione, accompanied by normalized basal and ACTHinduced adrenal steroid responses, suggests a possible role of ovarian androgens in adrenal steroidogenesis. This concept that some changes in adrenal steroidogenesis might be secondary to the ovarian hyperandrogenicity is supported by the findings that hyperoestrogenism and INS resistance may come from the extra-glandular aromatization of ovarian androgen excess and the direct impact of androgens on INS sensitivity (26).

Available data have also suggested a possible direct role of corticotrophin-releasing hormone (CRF)/ACTH in ovary physiology. It is believed that a human intraovarian CRF system may function as an inhibitory regulator for androgen biosynthesis within the thecal cell compartment, and is mediated through CRF receptors and its binding proteins (27). ACTH stimulation of $E_{2}$ and progesterone production from rat luteal cells has been defined to relate to its specific binding sites on cell surfaces and increased prostaglandin $\mathrm{E}_{2}$ production (28). In consideration of these factors, we performed adrenal dynamic tests for all subjects only during the mid-follicular phase of their menstrual cycles by using synthetic ACTH instead of CRF. Another possibility why adrenal dysfunction in patients with PCOS resolved after ovarian surgery in the present study may be the decreased LH levels. LH receptors have recently been identified in adult human reticularis and lower fasiculata cells, which are proven sites of adrenal androgen production (29). Human chorionic gonadotrophin, a structural and functional homologue of $\mathrm{LH}$, has been shown to stimulate adrenal androgen secretion in guinea pig adrenocortical cells (30). However, it seems unlikely as no obvious variations in both gonadotrophin levels were observed in these PCOS-affected women during this adrenal dynamic test.

As the number of patients in our pilot study is small and use of steroid ratios to estimate true enzyme activities should be interpreted with caution, the present results only suggest that ovarian function may influence adrenal androgen reserve in some highly selected cases with PCOS, mainly through $\Delta^{4}$ cytochrome $\mathrm{P} 450 \mathrm{c} 17 \alpha$ hyperactivity.

\section{References}

1 Carmina E, Koyama T, Chang L, Stanczyk FZ \& Lobo RA. Does ethnicity influence the prevalence of adrenal hyperandrogenism 
and insulin resistance in polycystic ovary syndrome? American Journal of Obstetrics and Gynecology 1992167 1807-1812.

2 Lobo RA \& Goebelsmann U. Adult manifestations of congenital adrenal hyperplasia due to incomplete 21-hydroxylase deficiency mimicking polycystic ovarian disease. American Journal of Obstetrics and Gynecology 1980138 720-724.

3 Rodin A, Thakkar H, Taylor N \& Clayton R. Hyperandrogenism in polycystic ovary syndrome. Evidence of dysregulation of 11 beta-hydroxysteroid dehydrogenase. New England Journal of Medicine 1994330 460-465.

4 Escobar-Morreale H, Pazos F, Potau N, Garcia-Robles R, Sancho JM \& Varela C. Ovarian suppression with triptorelin and adrenal stimulation with adrenocorticotropin in functional hyperadrogenism: role of adrenal and ovarian cytochrome P450c17 alpha. Fertility and Sterility 199462 521-530.

5 Martikainen H, Salmela P, Nuojua-Huttunen S, Perala J, Leinonen S, Knip M et al. Adrenal steroidogenesis is related to insulin in hyperandrogenic women. Fertility and Sterility 199666 564-570.

6 Ditkoff EC, Fruzzetti F, Chang L, Stancyzk FZ \& Lobo RA. The impact of estrogen on adrenal androgen sensitivity and secretion in polycystic ovary syndrome. Journal of Clinical Endocrinology and Metabolism 199580 603-607.

7 Golan A, Ron-el R, Herman A, Soffer Y, Weinraub Z \& Caspi E. Ovarian hyperstimulation syndrome: an update review. Obstetrics and Gynecological Survey 198944 430-440.

$8 \mathrm{Wu}$ XK, Su YH \& Zhang ZX. Wedge resection to improve insulin resistance in polycystic ovary syndrome: a study among Chinese women. British Journal of Obstetrics and Gynaecology 1996103 $822-825$.

$9 \mathrm{Wu}$ XK \& Su YH. Different responses of insulin, C-peptide, and testosterone to an oral glucose tolerance test in two groups of women with polycystic ovarian syndrome. Acta Obstetrica et Gynecologica Scandinavica 199675 166-169.

10 Azziz R, Bradley E Jr, Huth J, Boots LR, Parker CR Jr \& Zacur HA. Acute adrenocorticotropin-(1-24) (ACTH) adrenal stimulation in eumenorrheic women: reproducibility and effect of ACTH dose subject weight, and sampling time. Journal of Clinical Endocrinology and Metabolism 199070 1273-1279.

11 Legro RS, Finegood D \& Dunaif A. A fasting glucose to insulin ratio is a useful measure of insulin sensitivity in women with polycystic ovary syndrome. Journal of Clinical Endocrinology and Metabolism 199883 2694-2698.

12 Kaaijk EM, Beek JF, Hamerlynck JV \& van der Veen F. Unilateral oophorectomy in polycystic ovary syndrome: a treatment option in highly selected cases? Human Reproduction 199712 $2370-2372$

13 Emans SJ, Grace E, Woods ER, Mansfield J \& Crigler JF Jr. Treatment with dexamethasone of androgen excess in adolescent patients. Journal of Pediatrics 1988112 821-826.

14 Daly DC, Walters CA, Soto-Albors CE, Tohan N \& Riddick DH. A randomized study of dexamethasone in ovulation induction with clomiphene citrate. Fertility and Sterility 198441 844-848.

15 Gibson M, Lackritz R, Schiff I \& Tulchinsky D. Abnormal adrenal responses to adrenocorticotropic hormone in hyperandrogenic women. Fertility and Sterility 198033 43-48.

16 Gonzalez F, Chang L, Horab T, Stanczyk FZ, Crickard K \& Lobo RA. Adrenal dynamic responses to physiologic and pharmacologic adrenocorticotropic hormone stimulation before and after ovarian steroid modulation in women with polycystic ovary syndrome. Fertility and Sterility 199971 439-444.

17 Azziz R, Bradley EL Jr, Potter HD \& Boots LR. Adrenal androgen excess in women: lack of a role for 17-hydroxylase and 17,20-lyase dysregulation. Journal of Clinical Endocrinology and Metabolism 199580 400-405.

18 Unluhizarci K, Kelestimur F, Sahin Y \& Bayram F. The treatment of insulin resistance does not improve adrenal cytochrome P450c17alpha enzyme dysregulation in polycystic ovary syndrome. European Journal of Endocrinology 1999140 56-61.

19 Velazquez E, Acosta A \& Mendoza SG. Menstrual cyclicity after metformin therapy in polycystic ovary syndrome. Obstetrics and Gynecology $199790392-395$.

20 Morin-Papunen LC, Koivunen RM, Ruokonen A \& Martikainen HK. Metformin therapy improves the menstrual pattern with minimal endocrine and metabolic effects in women with polycystic ovary syndrome. Fertility and Sterility 199869 691-696.

21 Acby O \& Gundogdu S. Can metformin reduce insulin resistance in polycystic ovary syndrome? Fertility and Sterility 199665 946-949.

22 Ehrmann DA, Cavaghan MK, Imperial J, Sturis J, Rosenfield RL \& Polonsky KS. Effects of metformin on insulin secretion, insulin action, and ovarian steroidogenesis in women with polycystic ovary syndrome. Journal of Clinical Endocrinology and Metabolism 199782 524-530.

23 Hasegawa I, Murakawa H, Suzuki M, Yamamoto Y, Kurabayashi T \& Tanaka K. Effect of troglitazone on endocrine and ovulatory performance in women with insulin resistance-related polycystic ovary syndrome. Fertility and Sterility 199971 323-327.

24 Fruzzetti F, Melis GB, Mais V, Beconcini D, Paoletti AM, Cristiani G et al. High testosterone levels of ovarian origin affect adrenal steroidogenesis? Journal of Clinical Endocrinology and Metabolism $199172426-431$.

25 Carmina E, Gonzalez F, Vidali A, Stanczyk FZ, Ferin M \& Lobo RA. The contributions of oestrogen and growth factors to increased adrenal androgen secretion in polycystic ovary syndrome. Human Reproduction 199914 307-311.

26 Polderman KH, Gooren LJ, Asscheman H, Bakker A \& Heine RJ. Induction of insulin resistance by androgens and estrogens. Journal of Clinical Endocrinology and Metabolism $1994 \quad 79$ 265-271.

27 Erden HF, Zwain IH, Asakura H \& Yen SS. Corticotropinreleasing factor inhibits luteinizing hormone-stimulated P450c17 gene expression and androgen production by isolated thecal cells of human ovarian follicles. Journal of Clinical Endocrinology and Metabolism 199883 448-452.

28 Horvath E, Varga B \& Stark E. Action of ACTH in the luteal ovary. Acta Physiologica Hungarica 199178 69-76.

29 Pabon JE, Li X, Lei ZM, Sanfilippo JS, Yussman MA \& Rao CV. Novel presence of luteinizing hormone/chorionic gonadotropin receptors in human adrenal glands. Journal of Clinical Endocrinology and Metabolism 199681 2397-2400.

30 Fenske M. Saliva cortisol and testosterone in the guinea pig: measures for the endocrine function of adrenals and testes? Steroids $199661647-650$.

Received 14 December 1999

Accepted 30 May 2000 